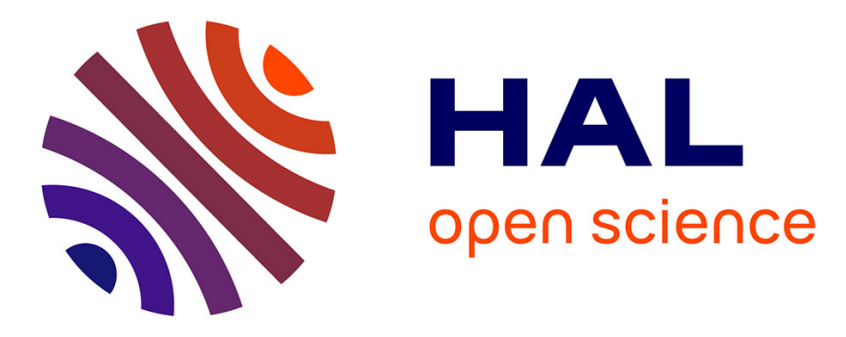

\title{
Control design for over-actuated systems based on reliability indicators
}

\author{
Ahmed Khelassi, Didier Theilliol, Philippe Weber
}

\section{To cite this version:}

Ahmed Khelassi, Didier Theilliol, Philippe Weber. Control design for over-actuated systems based on reliability indicators. UKACC International Conference on Control, CONTROL 2010, Sep 2010, Coventry, United Kingdom. pp.CDROM. hal-00520197

\section{HAL Id: hal-00520197 \\ https://hal.science/hal-00520197}

Submitted on 23 Sep 2010

HAL is a multi-disciplinary open access archive for the deposit and dissemination of scientific research documents, whether they are published or not. The documents may come from teaching and research institutions in France or abroad, or from public or private research centers.
L'archive ouverte pluridisciplinaire HAL, est destinée au dépôt et à la diffusion de documents scientifiques de niveau recherche, publiés ou non, émanant des établissements d'enseignement et de recherche français ou étrangers, des laboratoires publics ou privés. 


\title{
Control Design for over-actuated Systems based on Reliability Indicators
}

\author{
A. Khelassi, D. Theilliol, P. Weber \\ Centre de Recherche en Automatique de Nancy (CRAN) \\ CNRS UMR 7039, Nancy-Université \\ BP 70239, 54506 Vandoeuvre Cedex, France \\ (Tel: +33 383684 465; e-mail: ahmed.khelassi@cran.uhp-nancy.fr)
}

\begin{abstract}
This paper interests to the redundancy management of the over-actuated control systems. A new approach to manage the actuator redundancy, for the control allocation compared to the LQ method is proposed based on the reliability analysis. The aim is to preserve the health of the actuators and the availability of the system. The desired efforts and the control inputs are distributed and generated properly in order to maintain the system performance and preserve the actuators with a hight overall system reliability. The proposed approach is illustrated with a flight control application.
\end{abstract}

Keywords: Redundancy management, Control allocation, Optimal control, Reliability, Flight control.

\section{INTRODUCTION}

In order to respect the growing of economic demand for high plant availability, and system safety, dependability is becoming an essential need in the industrial automation. In this context and to satisfy these requirements, faulttolerant control (FTC) is introduced. The aim of FTC systems is to keep plant available by the ability to achieve the objectives that have been assigned to the system in the faulty behavior and accept reduced performance when critical faults occur (Blanke et al. (2006), Noura et al. (2009)). In most safety critical systems, the actuator redundancy is often used. For example, there are three major control effectors in aircraft flight control: aileron, elevator and rudder. They are usually designed using one control effector or actuator for each rational degree of freedom. However, due to the increased requirements on the reliability, maneuverability; and survivability of future aircraft, control effectors are no longer limited to these three conventional control effectors and many more control actuators have been introduced.

Several tools and approaches have been proposed and used to manage the redundancy and to distribute the control effort among a set of actuators. A common approach is to use the optimal control design to shape the closedloop dynamics as well as the actuator control distribution in one step. In particular, optimized methods like linear quadratic control (Kawakernaak and Sivan (1972)) and robust control (Gao and Antsaklis (1991)) are readily available.

An alternative issue is to separate the regulation task from the control distributing. Indeed, the control law specifies only the desired control efforts to be produced, and a separate control allocation module is introduced in the closed loop to distribute the control among the actuators (Harkegard (2003)). This strategy is used in several practical applications in aerospace control. In Durham (1993) and Bodson (2002), the control allocation is applied to several airplane flight. Bordignon (1996) illustrates this technique for an F-18 fighter with seven independent moments. A review of existing methods can be found in Enns (1998). The advantage of the control allocation strategy is the possibility to accommodate the faulty system without modify the controller parameters.

The main goal of FTC is to maintain the system stability and improve the overall system reliability in the event of such faults or failures (Zhang et al. (2007)). However, it is difficult to establish a functional linkage between the overall system reliability and control performance requirements (Theilliol et al. (2009)). Some works have introduced the reliability analysis for fault-tolerant control system in order to take into account the health of the degraded system in the reconfiguration strategy (Guenab et al. (2006)). The reconfigurability analysis has been investigated for a reliable fault-tolerant control design in Khelassi et al. (2009). In this paper, the over-actuated control is proposed based on the reliability analysis and applied on linearized model aircraft. The aim is to improve the global system reliability during the mission. The integration of reliability indicators on linear optimal control (LQ) for over-actuated system is proposed and compared to the allocation strategy, where the connexion of theses approaches is published in the work of Harkegard and Glad (2004). The weighing matrices of these two strategies are considered as a key to generate a specific control inputs improving the availability of the system.

The paper is organized as follows: control allocation issue, and problem formulation are presented in Section II. The integration of reliability indicators in the control allocation strategy is proposed for a reliable controller. In Section III, the solution for a reliable control allocation strategy is presented where the weighing matrix is synthesis 
based on the reliability indicators. The contribution to a linear quadratic controller that preserves the actuators and improves the overall system reliability is proposed. The proposed approach for control allocation is illustrated and applied to a linearized aircraft model from ADMIR simulator in Section IV. Finally, concluding remarks are given in the last part of this work.

\section{CONTROL ALLOCATION BASED ON RELIABILITY INDICATORS}

\subsection{Problem statement}

Let us consider the linearized dynamic of the aircraft given by:

$$
\begin{aligned}
& \dot{x}(t)=A x(t)+B_{u} u(t) \\
& y(t)=C x(t)
\end{aligned}
$$

where $A \in \mathbb{R}^{n \times n}, B_{u} \in \mathbb{R}^{n \times m}$ and $C \in \mathbb{R}^{p \times n}$ are respectively, the state, the control and the output matrices. $x \in \mathbb{R}^{n}$ is the system state, $u \in \mathbb{R}^{m}$ is the control input, $y \in \mathbb{R}^{p}$ is the system output, and $\left(A, B_{u}\right)$ is stabilizable. Control allocation is generally used for overactuated systems, where the number of operable control is greater than the controlled variables. Let us assume that $\operatorname{rank}\left(B_{u}\right)=k<m$. This implies that $B_{u}$ can be factorized as:

$$
B_{u}=B_{v} B
$$

where $B_{v} \in R^{n \times k}$ and $B \in \mathbb{R}^{k \times m}$. An alternative description of (1) can be given as:

$$
\begin{aligned}
& \dot{x}(t)=A x(t)+B_{v} v(t) \\
& v(t)=B u(t) \\
& y(t)=C x(t)
\end{aligned}
$$

where $v \in \mathbb{R}^{k}$ is the virtual control input, called as the total control effort produced by the actuators and defined by the controller. For simplicity and for this study, the case $k=p$ is considered, i.e., when the number of virtual control equals the number of outputs to be controlled.

The control allocation problem can be expressed as a constrained linear mapping problem,

$$
\begin{gathered}
v(t)=B u(t) \\
u_{\min } \leq u \leq u_{\max }
\end{gathered}
$$

where (4) is the physical actuators saturation.

Optimized based control allocation methods aim to find an optimal solution: pick the best one if there are several solutions, or search for a feasible one such that $B u(t)$ approximates $v(t)$ well as possible when there is no exact solution. The optimal control input can be obtained by a two-step optimization, namely sequential quadratic programming:

$$
\begin{aligned}
& \psi=\underset{u_{\min } \leq u \leq u_{\max }}{\arg \min }\|B u-v\|_{2} \\
& u=\arg \min _{u \in \psi}\left\|W_{u}\left(u-u_{d}\right)\right\|_{2}
\end{aligned}
$$

where $u_{d}$ is the desired control input and, $W_{u} \in \mathbb{R}^{m \times m} \succ$ 0 is a weighting matrix used to give some priority level to the actuators.

In order to manage more effectively the redundant actuators, a specific choice of the weighting matrix $W_{u}$ is proposed based on actuators reliability indicators. The weighing matrix $W_{u}$ is considered as a key to manage the redundant actuators and contribute to a reliable controller design. This technique can increase the life time of the system and prevent additional faults from occurring.

\subsection{Control allocation for a reliable controller}

In general, the reliability is defined as the probability that units, components, equipments and systems will accomplish its intended function for a specified period of time under some stated conditions and specific environments (Gertsbakh (2000)).

In many situations and especially in the considered study, failure rates are obtained from components under different levels of loads. Several mathematical models have been developed to define the failure level in order to estimate the failure rate $\lambda$ (Martorell et al. (1999)). Proportional hazard model introduced by Cox (1972) is used in this paper.

Definition 1. The failure rate is modeled as follows:

$$
\lambda_{i}=\lambda_{i}^{b l} \times g_{i}(\ell, \vartheta)
$$

where $\lambda_{i}^{b l}$ represents the baseline failure rate (nominal failure rate) for the $i^{\text {th }}$ subsystem or component and $g_{i}(\ell, \vartheta)$ is a function (independent of time) taking into account the effects of applied loads with $\ell$ presenting an image of the load and $\vartheta$ defining some parameters of the subsystem or component.

Definition 2. Different definitions of the load function $g_{i}(\ell, \vartheta)$ exist in the literature (Martorell et al. (1999)). However, the exponential form is commonly used (Dale (1985)). Moreover, the failure rate functions for the exponential distribution change according to the load level, which are assumed to be directly associated to the control input.

$$
g_{i}(\ell, \vartheta)=g\left(\left\|u_{i}\right\|\right)=e^{f\left(u_{i}\right)}
$$

where $f($.$) is an increased monotonic function.$

Definition 3. For the exponential distribution of reliability, the mean time before the first failure (MTTF) can be adopted as a reliability indicator defined such as:

$$
\operatorname{MTTF}=\int_{0}^{\infty} R(t) d t=\frac{1}{\lambda}
$$

Moreover, for ' $m$ ' redundant components, the overall system reliability can be computed at the end of the mission $t=t_{M}$ as follows:

$$
R_{g}\left(t_{M}\right)=1-\prod_{i=1}^{m}\left(1-R_{i}\left(t_{M}\right)\right)
$$

In the order to decelerate the actuators degradation, the desired efforts in the control allocation will be distributed to the different actuators taken into account their reliability characteristics. As given in (7), the failure rate of the actuator is defined according to the load level which is proportional to the control input applied, where

$$
\lambda_{i} \geq \lambda_{i}^{b l}
$$

Moreover, the optimal control input $u^{*}=\left(u_{1}^{*}, u_{2}^{*}, \ldots, u_{m}^{*}\right)$ of the control allocation problem (3) and (4) is defined according the values of the weighing matrix $W_{u}$, where 


$$
u^{*}=\underset{u_{\min } \leq u \leq u_{\max }}{\arg \min }\left\|W_{u} u\right\|_{2}
$$

Since $W_{u}=\operatorname{diag}\left\{w_{1} \ldots, w_{m}\right\} \succ 0$ is a definite diagonal matrix, the following relation can be obtained:

$$
\left.\begin{array}{l}
w_{i} \rightarrow \infty: u_{i}^{*} \rightarrow \epsilon \\
w_{i} \rightarrow \epsilon: u_{i}^{*} \rightarrow u_{\max }
\end{array}\right\}
$$

To perform the solution of the control allocation problem, and keep set of the actuators available as long as possible, the desired efforts computed from the control law can be distributed proportionally to the actuators reliability as follows:

$$
W_{u}=\left(\begin{array}{cccc}
\frac{\lambda_{1}^{b l}}{\lambda_{\max }^{b l}} & & & 0 \\
& \frac{\lambda_{2}^{b l}}{\lambda_{\max }^{b l}} & & \\
& & \ddots & \\
0 & & & \frac{\lambda_{m}^{b l}}{\lambda_{\max }^{b l}}
\end{array}\right) \succ 0
$$

where $\lambda_{\max }^{b l}=\max \left(\lambda_{i}^{b l}\right), i=1: m$. The actuators are used in the efforts distribution proportionally to their reliabilities. This off line synthesis of the control allocation strategy less damage the sensible actuators which, improve the system availability and the actuators healthy.

Indeed, for the proposed weighting matrix design $W_{u}(13)$, the following relations can be achieved:

$$
\left.\begin{array}{l}
\lambda_{i}^{b l} \rightarrow \lambda_{\max }^{b l}: u_{i}^{*} \rightarrow \epsilon \\
\lambda_{i}^{b l} \rightarrow \lambda_{\min }^{b l}: u_{i}^{*} \rightarrow u_{\max }
\end{array}\right\}
$$

where $\lambda_{\min }^{b l}$ is the failure rate of the most reliable actuator.

\section{SOLUTIONS FOR RELIABLE CONTROL OF OVER-ACTUATED SYSTEMS}

The control design for over-actuated dynamic systems can be performed in two fundamentally different ways, either a separate control allocator is used, or the control allocation is included in the control-loop. Based on the work of Harkegard and Glad (2004), the connexion of the two strategies for linear quadratic design is proved. For the control allocation strategy, the control input $u$ is allocated to the different actuators depending on the weight matrix $W_{u}$. In this section, the optimal control $u$, solution of the problem (5) and (6) is allocated smartly, where $W_{u}$ is chosen according to the actuators reliability indicators (13). Moreover, a contribution to a reliable LQ controller is proposed where, the control law is synthesis with a specific choice of the weighting matrices based on the actuators reliability indicators.

\subsection{Pseudo-inverse method for control allocation}

The control allocation problem has been extensively studied in recent years and several numerical strategies have been proposed in fault-free case and solved off-line (Bodson (2002), Harkegard (2003)).

if the above control constraint (4) is not considered, an explicit solution can be obtained from minimization of the above quadratic problem (5),(6) as follows:

$$
\begin{aligned}
& \min _{u} J=\left\|W_{u} u\right\| \\
& s . q \quad B u(t)=v(t)
\end{aligned}
$$

and the solution is given based on a weighted pseudoinverse as follows:

$$
\begin{aligned}
& u(t)=P v(t) \\
& P=W_{u}^{-1}\left(B W_{u}^{-1}\right)^{+}
\end{aligned}
$$

where $(+)$ is the pseudo-inverse operator.

Obviously, there is no guarantee that the solution will not exceed the constraints. Improved approaches have been proposed to accommodate to the limits. The Redistributed pseudoinverse method (RPI) proposed by Virnig and Bodden (1994) is an alternative solution, in which all control inputs that violate their bounds in the pseudoinverse solution are saturated and removed from the optimization. Then the control problem is resolved with only the remaining control inputs as free variables. The Cascaded Generalized Inverse (CGI) method proposed by Bordignon (1996) is an iterative redistributed pseudoinverse. All control inputs that violate their bounds are set saturated values and removed at each step, and the redistribution process is continued until either the pseudoinverse solution is feasible or all control inputs are saturated. Enns (1998) also suggests to compute the pseudoinverse solution iteratively, as in CGI but to only saturate one control input per iteration. For the proposed approach, the most reliable actuator is saturated at each step and removed from the CGI optimization problem.

\subsection{Active set method for constrained control allocation}

While the actuators saturation are considered, a common technique is to approximately reformulate the sequential optimization problem Eq.(5) and Eq.(6) as a mixed optimization problem as follows:

$$
u^{*}=\underset{u_{\min } \leq u \leq u_{\max }}{\arg \min }\|B u-v\|_{2}+\gamma\left\|W_{u}\left(u-u_{d}\right)\right\|_{2}
$$

where $\gamma>>1$. In fact, the Weighted Least Squares algorithm based on the set active method Harkegard and Glad (2004) can be used to found a good approximation of the virtual control input.

The bound and equality constrained least squares problem may write as follows:

$$
\begin{gathered}
u=\arg \min _{u}\|\bar{A} u-\bar{b}\|_{2} \\
B u=v \\
\bar{C} u \geq U
\end{gathered}
$$

where $\bar{C}=\left[\begin{array}{l}I \\ -I\end{array}\right]$ and $U=\left[\begin{array}{l}u_{\min } \\ -u_{\max }\end{array}\right]$.

The active set method solves this problem by solving a series of equality constraints problem. Indeed, the control allocation problem (16) can be written as the following cost function:

$\|B u-v\|_{2}+\gamma\left\|W_{u}\left(u-u_{d}\right)\right\|_{2}=\left\|\left(\begin{array}{c}\gamma^{\frac{1}{2}} B \\ W_{u}\end{array}\right) u-\left(\begin{array}{l}\gamma^{\frac{1}{2}} v \\ W_{u} u_{d}\end{array}\right)\right\|^{2}$

This problem is equivalent to the constrained least squares problem (17), where the optimal control input can be found as following: 
Let $u^{0}$ be a feasible starting point, satisfying the constraints (18). Let the working set $\mathcal{W}$ contain the active inequality constraints at $u^{0}$. Given a sub-optimal iterate $u^{i}, i=1,2, \ldots$, find the optimal perturbation $p$ considering the inequality constraints in the working set as equality constraints and disregarding the remaining inequality constraints. Solve

$$
\begin{gathered}
\min _{p}\left\|\bar{A}\left(u^{i}+p\right)-\bar{b}\right\| \\
B p=0 \\
p_{i}=0, i \in \mathcal{W}
\end{gathered}
$$

For one situation, if $u^{i}+p$ is feasible, set $u^{i+1}=u^{i}+p$ and compute the Lagrange multipliers in the following form

$$
\bar{A}^{T}(\bar{A} u-\bar{b})=\left(B^{T} \bar{C}_{0}^{T}\right)\left(\begin{array}{c}
\mu \\
\psi
\end{array}\right)
$$

where $\bar{C}_{0}$ contains the rows of $\bar{C}$ that correspond to constraints in the working set. $\mu$ and $\psi$ are associated to with the active constraints in (18).

In fact, if all $\psi \geq 0, u^{i+1}$ is the optimal solution. Iteration will stop with $u=u^{i+1}$, else, remove the constraints associated with the most negative $\psi$ from the working set. However, for another situation, if $u^{i}+p$ is infeasible, the maximum step $\alpha$ length should be determined such that $u^{i+1}=u^{i}+\alpha p$ is feasible. Then the bounding constraints at $u^{i+1}$ is added to the working set.

\subsection{Reliable $L Q$ control for over-actuated systems}

Consider the over-actuated system description (1), the standard LQ design is used to achieve the stability as well to distribute the control effort among the control inputs.

Design 1. The control input $u(t)$ is determined by solving $\min _{u} \int_{0}^{\infty}\left(\left(x-x^{*}\right)^{T} Q_{1}\left(x-x^{*}\right)+\left(u-u^{*}\right)^{T} R_{1}\left(u-u^{*}\right)\right) d t$ where $Q_{1} \succeq 0, R_{1} \succ 0$, and $x^{*}, u^{*}$ solve

$$
\begin{array}{r}
\min _{x, u} u^{T} R_{1} u \\
\text { subject to } A x+B_{u} u=0 \\
C x=r
\end{array}
$$

Hence, if there are several solution of $u$ that achieve $\dot{x}$ and $y=r$, the control input $u$ that minimizes $u^{T} R_{1} u$ is chosen. The control law is given by the following theorem,

Theorem 1. The optimal control law for Design 1 is given by

$$
\begin{aligned}
& u(t)=G r(t)-K x(t) \\
& G=R_{1}^{-\frac{1}{2}}\left(G_{0} R_{1}^{-\frac{1}{2}}\right)^{+} \\
& K=R_{1}^{-1} B_{u}^{T} S_{1}
\end{aligned}
$$

where

$$
G_{0}=C\left(B_{u} L-A\right)^{-1} B_{u}
$$

and $S_{1}$ is the unique positive semidefinite and symmetric solution to the algebraic Riccati equation

$$
A^{T} S_{1}+S_{1} A+Q_{1}-S_{1} B_{u} R_{1}^{-1} B_{u}^{T} S_{1}=0
$$

However, for the control allocation strategy, a stabilizing virtual control law $v(t)$ is designed here based on the LQ scheme and then, the $l_{2}$-optimal control allocation is used to distribute the control effect among the individual control inputs.

Design 2. The virtual control input $v(t)$ is determined by solving

$$
\min _{u} \int_{0}^{\infty}\left(\left(x-x^{*}\right)^{T} Q_{2}\left(x-x^{*}\right)+\left(v-v^{*}\right)^{T} R_{2}\left(v-v^{*}\right)\right) d t
$$

where $Q_{2} \succeq 0, R_{2} \succ 0$, and $x^{*}, v^{*}$ solve

$$
\begin{aligned}
A x+B_{u} u & =0 \\
C x & =r
\end{aligned}
$$

Then, the control input $u(t)$ is computed and generated by solving the control allocation problem (15)

Theorem 2. The optimal control law for Design 2 is given by (16), where the optimal control input $v(t)$ is given following the theorem 1 ,

$$
v(t)=G r(t)-K x(t)
$$

Here, the expression of $G$ and $K$ are computed as in theorem 1 according to the matrices $Q_{2}$ and $R_{2}$.

The connexion between Design 1 and Design 2 is the subject of the next theorem.

Theorem 3. The control laws generated by Designs 1 and 2 are the same for the following choices:

- Given $Q_{1}$ and $R_{1}$,

$$
\begin{aligned}
& Q_{2}=Q_{1} \\
& R_{2}=\left(B R_{1}^{-1} B^{T}\right)^{-1} \\
& W_{u}=R_{1}^{\frac{1}{2}}
\end{aligned}
$$

- Given $Q_{2}$ and $R_{2}$,

$$
\begin{aligned}
& Q_{1}=Q_{2} \\
& R_{1}=W_{u}^{2}+B^{T}\left(R_{2}-\left(B W_{u}^{-2} B^{T}\right)^{-1}\right) B
\end{aligned}
$$

Proof 1. . See, Harkegard (2003).

For a reliable LQ control design and in order to improve the safety and the overall system reliability, the weighting matrices are given by (25) where $W_{u}$ is chosen according to the reliability indicators(12).

\section{FLIGHT CONTROL EXAMPLE}

The ADMIRE model has been used by several researchers (e.g. Harkegard (2003))and within the Group of Aeronautical Research ans Technology in Europe (GARTEUR). The linear model used here has been obtained at a low speed flight condition of Mach 0.22 at an altitude of $3000 \mathrm{~m}$ and is similar to the one in Harkegard and Glad (2004). The states are $x=\left[\begin{array}{lllll}\alpha & \beta & p & q & r\end{array}\right]^{T}$ with controlled output $y=[\alpha \beta p]$; where $\alpha$ is the angle of attack $(\mathrm{rad}), \beta$ is the sideslip angle $(\mathrm{rad})$, and $p$ is the roll rate $(\mathrm{rad} / \mathrm{s}), q$ defines the pitch rate $(\mathrm{rad} / \mathrm{s})$ and $r$ is the yaw rate $(\mathrm{rad} / \mathrm{s})$. The control surface are $\sigma=\left[\begin{array}{llll}\sigma_{c} & \sigma_{r e} & \sigma_{l e} & \sigma_{r}\end{array}\right]^{T}$, which represent the deflections (rad) of the canard, right eleven, left eleven and rudder respectively. A linearized model Harkegard and Glad (2004) is :

$$
A=\left[\begin{array}{ccccc}
-0.5432 & 0.0137 & 0 & 0.9778 & 0 \\
0 & -0.1179 & 0.2215 & 0 & -0.9661 \\
0 & -10.5128 & -0.9967 & 0 & 0.6176 \\
2.6221 & -0.0030 & 0 & -0.5057 & 0 \\
0 & 0.7075 & -0.0939 & 0 & -0.2127
\end{array}\right]
$$




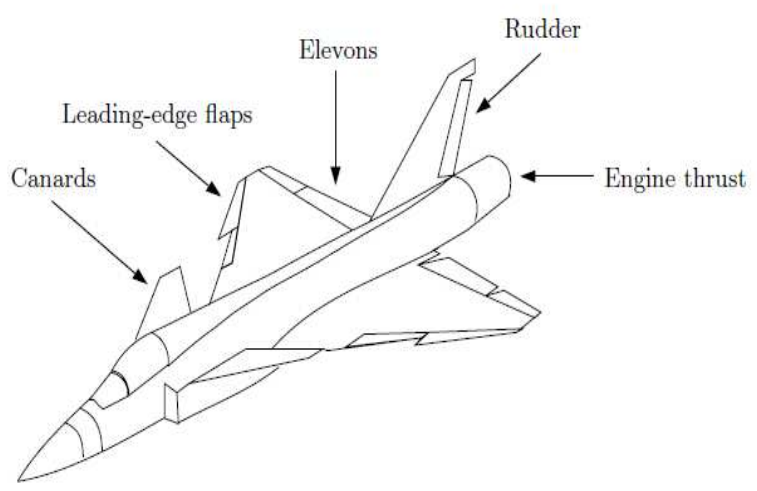

Fig. 1. Aircraft configuration

$$
B=\left[\begin{array}{cccc}
0.0069 & -0.0866 & -0.0866 & 0.0004 \\
0 & 0.0119 & -0.0119 & 0.0287 \\
0 & -4.2423 & 4.2423 & 1.4871 \\
1.6532 & -1.2735 & -1.2735 & 0.0024 \\
0 & -0.2805 & 0.2805 & -0.8823
\end{array}\right],
$$

In this example, the actuator dynamics are neglected, and the approximate model can be given where:

$$
B_{u}=B_{v} B
$$

and where

$$
\begin{gathered}
B=\left[\begin{array}{l}
0_{2 \times 3} \\
I_{3 \times 3}
\end{array}\right], \\
B=\left[\begin{array}{cccc}
0 & -4.2423 & 4.2423 & 1.4871 \\
1.6532 & -1.2735 & -1.2735 & 0.0024 \\
0 & -0.2805 & 0.2805 & -0.8823
\end{array}\right],
\end{gathered}
$$

Table 1. Failure rates of elementary components

\begin{tabular}{|c|c|}
\hline \multicolumn{2}{|c|}{ Failure rates } \\
\hline$\lambda_{1}^{b l}$ & $1 \mathrm{e}-2 \mathrm{~min}^{-1}$ \\
\hline$\lambda_{2}^{b l}$ & $8 \mathrm{e}-3 \mathrm{~min}^{-1}$ \\
\hline$\lambda_{3}^{b l}$ & $5 \mathrm{e}-3 \mathrm{~min}^{-1}$ \\
\hline$\lambda_{4}^{b l}$ & $9 \mathrm{e}-3 \mathrm{~min}^{-1}$ \\
\hline
\end{tabular}

The resulting virtual control input $v(t)$, contains the angular accelerations in roll, pitch, and yaw produced by the control surfaces.

To illustrate the proposed approach in the short time window, the values of the actuators failure rates are adapted with the simulated scenario in the example. In fact, the failure rates are considered with a very huge value and given in the table.1. To model the effects of the applied load (8) and evaluate the actuators degradation, the rootmean-square (RMS) of the control input applied for each actuator during the mission is considered. In fact, the RMS values are seen as an image on the average applied load for the deferent actuators.

In this example, the following control strategies are investigated. First the $l_{2}$ optimal control allocation for the approximate model is simulated and compared to the equivalent LQ design for the proposed weighing matrix $W_{u}=\operatorname{diag}(10,10,10,10)$ considered in Harkegard and Glad (2004). Then, the desired efforts are distributed against an optimal choice of the $W_{u}^{*}$ as given in (12). An optimal solution of the control allocation based on the reliability indicators is obtained in order to improve the availability of the system and preserve the actuators. The
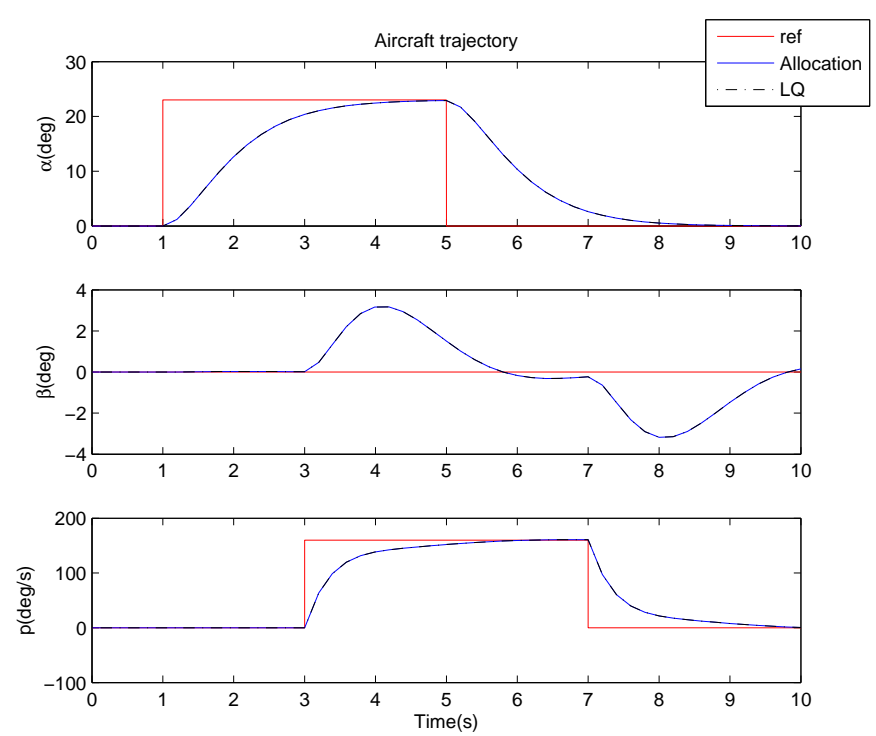

Fig. 2. Aircraft trajectory
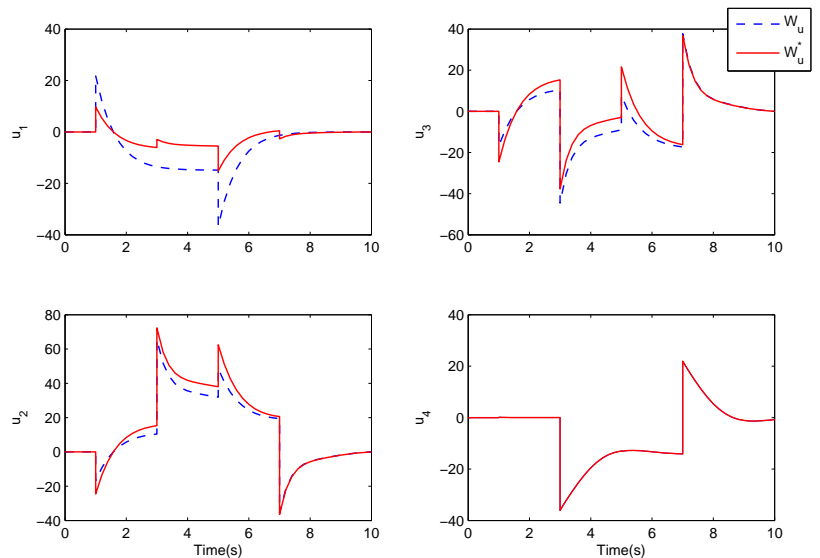

Fig. 3. Control inputs

applied optimal control inputs are compared to the the previous case.

$$
W_{u}^{*}=\left(\begin{array}{cccc}
1 & & \ldots & 0 \\
& 0.8 & & \vdots \\
\vdots & & 0.5 & \\
0 & \ldots & & 0.9
\end{array}\right)
$$

The figure(2) shows the simulation results when the actuator constraints are not included in the control problem. Since no saturation occur, design.1 and 2 give the exact same results.

The figure(3) shows the control inputs generated by the allocation module for an optimal efforts distribution. these optimal control inputs are compared to the arbitrary choice. The desired efforts for the proposed approach is distributed with respect to the reliability of each actuators where, the most reliable actuator is more solicited in the control allocation. The overall system reliability is evaluated in figure(4) for the different choices of the weighting matrix. In the proposed approach, the distribution of the 
Table 2. Applied load of the actuators

\begin{tabular}{|c||c|c|c|c|}
\hline & $\lambda_{1}^{b l}$ & $\lambda_{2}^{b l}$ & $\lambda_{3}^{b l}$ & $\lambda_{4}^{b l}$ \\
\hline$R M S$ & 1.5388 & 1.6560 & 3.0701 & 1.5862 \\
\hline$R M S^{*}$ & 0.7242 & 1.4859 & 3.6607 & 1.5862 \\
\hline
\end{tabular}

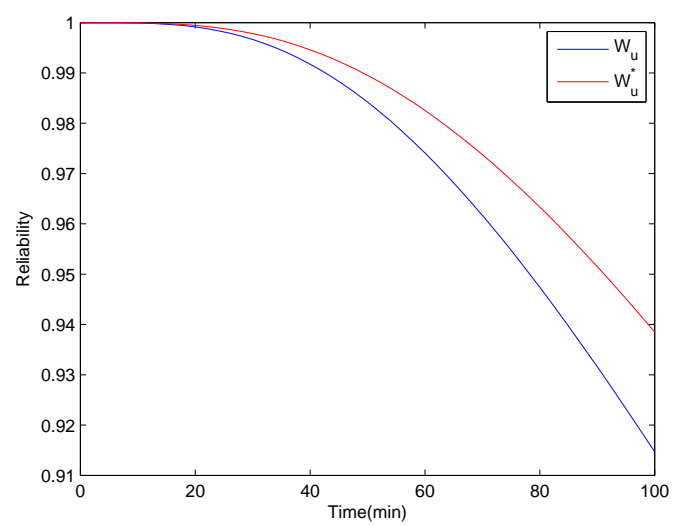

Fig. 4. Overall system reliability

desired efforts is achieved with a high overall system reliability. This technique preserve the actuators and can improve the availability of the pant where, the control inputs is applied taken into account the reliability characteristic and the degradation state of the actuators. The table. 2 shows the evolution of the considered applied load according to the energy consumption for each actuator. For the proposed approach, the actuators are requested in the control allocation due to their baseline failure rate where the less sensible actuator is less used in the efforts distribution.

\section{CONCLUSION}

A contribution for an optimal strategy of control allocation is proposed. The distribution of the desired efforts is considered based on the actuators reliability. This strategy guarantee the distribution of the desired efforts with a hight overall system reliability. The control inputs are applied to the system taken into account the health of the actuators which, can preserve the actuators and improve the overall system reliability. A contribution to a reliable LQ controller is presented where the connexion between the control allocation strategy and LQ control design is illustrated.

\section{REFERENCES}

M. Blanke, M. Kinnaert, J. Lunze, and M. Staroswiecki. Diagnosis and fault tolerant control. Control Systems Series, Springer-Verlag London, 2006.

M. Bodson. Evaluation of optimization methods for control allocation. Journal of Guidance, Control, and Dynamics, 25:703-711, 2002.

K.A. Bordignon. Constrained control allocation for systems with redundant control effector. $\mathrm{PhD}$ thesis, Vergenia Polytechnic Institute and State University, 1996.

D.R. Cox. Regression models and life tables. JR Stat Soc, 34:187-220, 1972.

J.C. Dale. Application of the proportional hazards model in the reliability field. Reliability Engineering, 10:1-14, 1985 .
W.C. Durham. Constrained control allocation. Journal of Guidance, Control, and Dynamics, 16:717-125, 1993.

D. Enns. Control allocation appraochs. AIAA Guidance, Navigation, and Control Conference, pages 98108, 1998. VA, USA.

Z. Gao and P. Antsaklis. Stability of the pseudo-inverse method for reconfigurable control systems. International Journal of Control, pages 717-729, 1991.

I. Gertsbakh. Reliability theory with applications to preventive maintenance. Springer, 2000.

F. Guenab, D. Theilliol, P. Weber, Y.M. Zhang, and D. Sauter. Fault tolerant control desing: A reconfiguration strategy based on reliability analysis under dynamic behaviour constraints. In Proc. of 6th IFAC symposium Safeprocess, 2006. Beijing, RP China.

O. Harkegard. Backstepping and Control Allocation with Applications to Flight Control. PhD thesis, Department of Electrical Engineering Linkoping University, Sweden, 2003.

O. Harkegard and S.T. Glad. Resolving actuator redundancy-optimal control vs. control allocation. 41: 137-144, 2004.

H. Kawakernaak and R. Sivan. Linear Optimal Control Systems. New York, Wiley-Interscience, 1972.

A. Khelassi, D. Theilliol, and P. Weber. Reconfigurability for reliable fault-tolerant control design. $7^{\text {th }}$ Workshop on Advanced Control and Diagnosis, 2009. Zielon Gora, Poland.

S. Martorell, A. Sanchez, and V.Serradell. Age-dependent reliability model considering effects of maintenance and working conditions. Reliability Engineering and System Safety, 64:19-31, 1999.

H. Noura, D. Theilliol, J.C. Ponsart, and A. Chamssedine. Fault tolerant control systems: Design and practical application. Springer Dordrecht Heidelberg London, 2009.

D. Theilliol, A. Chamssedine, Y. Zhang, and P. Weber. Optimal reconfigurable control allocation design based on reliability analysis. $7^{\text {th }}$ IFAC Symposium on Fault Detection, Supervision and Safety of Technical Processes, 2009. Barcelona, Spain.

J.C. Virnig and D.S. Bodden. Multivariable control allocation and control law conditioning when control effectors limits. AIAA Guidance, Navigation, and Control Conference, pages 572-582, 1994. Scottsdale, AZ.

Y. Zhang, V.S. Suresh, B. Jiang, and D. Theilliol. Reconfigurable control allocation against aircraft control effector failures. IEEE International Conference on Control Applications, CCA 2007, 2007. Singapore. 